\title{
Amiodarone in cardiothoracic surgery patients: What is a surgeon to do?
}

William L. Holman, MD

See related article on page 633 .

From the Department of Surgery, University of Alabama at Birmingham, Birmingham, Ala.

Received for publication June 24, 2002; accepted for publication July 19, 2002.

Address for reprints: William L. Holman, MD, the Department of Surgery, University of Alabama at Birmingham, 703 S 19th St, Room 719, Birmingham, AL 35294-0007.

J Thorac Cardiovasc Surg 2003;125:463-4

Copyright $\odot 2003$ by The American Association for Thoracic Surgery

$0022-5223 / 2003 \$ 30.00+0$

doi: $10.1067 / \mathrm{mtc} .2003 .10$

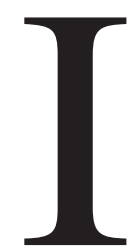

$\mathrm{n}$ this issue of The Journal of Thoracic and Cardiovascular Surgery, the CAMIAT Investigators ${ }^{1}$ present an interesting post hoc analysis of amiodarone used in 82 patients undergoing cardiac surgery who were part of a larger trial that examined prophylactic amiodarone therapy in patients at risk for sudden death after myocardial infarction. This post hoc analysis has a problem common to all such analyses; namely the initial research proposal was not specifically designed or powered to answer the questions that were raised in the secondary analysis. Nevertheless, the small number of prospective trials (especially prospective randomized trials) of amiodarone used in the setting of cardiac surgery make this information useful for placing the risks of amiodarone into perspective with its benefits.

Crystal and associates found no differences in the 7- and 30-day mortalities, duration of intensive care unit stay, risk of atrial or ventricular fibrillation, and risk of pulmonary complications between those who did and did not receive amiodarone before a cardiac operation. However, there was a higher rate of postoperative intra-aortic balloon pump use among the patients treated with amiodarone. Thus this study supports the notion that amiodarone has a negative inotropic or vasodilatory effect but refutes other commonly held beliefs regarding the drug's adverse effects on heart rate and pulmonary function. It should also be noted that the doses of amiodarone were relatively low, and the median time between cessation of amiodarone dosing and surgery was 15 days in this trial.

I remember many years ago discussing the perioperative use of amiodarone with a friend from Europe. He believed that the side effects of amiodarone were greatly outweighed by its efficacy. I disagreed and thought that amiodarone would never be widely used in the United States because of its acute and chronic toxicities. It is time for me to "eat crow." Amiodarone is currently the most commonly prescribed antiarrhythmic drug in Europe, Latin America, and North America, and its use increased by $20 \%$ from 1997 to $1998 .^{2}$ As pointed out by the CAMIAT Investigators, the issue of how to manage patients receiving short- or long-term amiodarone therapy is one that surgeons must face on a regular basis. An alternative approach to operating on patients fully loaded with amiodarone is to defer surgery until amiodarone has been eliminated from the system. ${ }^{3}$ For most patients this is impractical because of the large volume of distribution and consequent long elimination half-life of amiodarone ( 30 to 60 days), as well as because of the threat of arrhythmia recurrence while amiodarone levels are falling. It is timely to reflect on what have we learned during the past decade and define as best we can the risks for patients receiving amiodarone therapy who face a cardiac (or pulmonary) surgical procedure.

The answers presented in this editorial are based on evolving information that is by definition incomplete. Moreover, amiodarone is typically used in patients with life-threatening arrhythmias in the setting of important heart disease. Dissecting out the toxic effect of amiodarone from the morbidity and mortality related to the disease process itself is difficult. This difficulty suggests that we should rely more heavily on information derived from prospective randomized trials (even if it comes from a secondary analysis of the data), rather than information from any other source.

Acute postoperative pulmonary toxicity associated with amiodarone therapy and 
exposure to high concentrations of inspired oxygen appears to be a sporadic event that usually follows an inexorably worsening course culminating in death. ${ }^{4}$ The evidence that this occurs and is related to oxygen dose is based on animal studies and clinical observations. Fortunately, limiting inspired oxygen concentration during and after surgery appears to ameliorate this complication. Vigilant care of patients in the current era may explain why this problem was not found in the CAMIAT analysis or in another post hoc analysis of a prospective randomized trial. ${ }^{5}$

Other adverse events, such as low cardiac output or hypotension requiring perioperative intra-aortic balloon counterpulsation, may occur more commonly among patients treated with amiodarone, particularly if the therapy is long term and high dose. However, the mortality associated with this morbidity appears low according to limited information from prospective randomized trials as well as retrospective studies that account for the influence of the patients' underlying heart disease. ${ }^{5,6}$ This observation is made recognizing the limitation of extrapolating from relatively small study groups to accurately define the etiology for adverse events in a group of patients with many potential reasons for complications.

Another issue to consider is the prophylactic use of amiodarone to minimize the occurrence of postoperative atrial fibrillation. Several studies have shown that amiodarone can decrease the prevalence of postoperative atrial fibrillation, although the details of optimal dosing and duration of treatment have not yet been worked out. ${ }^{7}$ Toxicity of amiodarone was not found to be a problem in these studies, possibly because the dosage was relatively low and the drug was given for a short period of time. Thus it seems that the cost of this prophylaxis and its efficacy in relationship to alternatives for prevention of atrial fibrillation, such as $\beta$-antagonists, are more important questions to answer than questions of toxicity.

Cardiac surgery is a dynamic specialty. We develop novel therapies, incorporate useful ideas and materials from other fields, and respond to the constantly changing challenges posed by our patients. The typical cycle for responding to challenge or change is one of recognition, skepticism and observation, further evaluation, and eventually consensus. There is not yet consensus regarding the issue of risks and benefits of amiodarone therapy in cardiac surgery, but there is mounting information to indicate that amiodarone is not as hazardous as was initially thought. With appropriate vigilance, it can be used with a reasonable degree of safety to provide an important antiarrhythmic benefit.

\section{References}

1. Crystal E, Kahn S, Roberts R, Thorpe K, Gent M, Cairns JA, et al. Long-term amiodarone therapy and the risk of complications after cardiac surgery: results from the Canadian Amiodarone Myocardial Infarction Arrhythmia Trial (CAMIAT). J Thorac Cardiovasc Surg. 2003;125:633-7.

2. Connolly SJ. Evidence-based analysis of amiodarone efficacy and safety. Circulation. 1999;100:2025-34.

3. Mickleborough LL, Maruyama H, Mohamed S, Rappaport DC, Downar E, Butany $\mathrm{J}$ et al. Are patients receiving amiodarone at increased risk for cardiac operations? Ann Thorac Surg. 1994;58:622-9.

4. Kay GN, Epstein AE, Kirklin JK, Diethelm AG, Graybar G, Plumb VJ. Fatal postoperative amiodarone pulmonary toxicity. Am J Cardiol. 1988;62:490-2.

5. Singh SN, Fisher SG, Deedwania PC, Rohatgi P, Singh BN, Fletcher RD. Pulmonary effect of amiodarone in patients with heart failure. The Congestive Heart Failure-Survival Trial of Antiarrhythmic Therapy (CHF-STAT) Investigators (Veterans Affairs Cooperative Study No. 320). J Am Coll Cardiol. 1997;30:514-7.

6. Rady MY, Ryan T, Starr NJ. Preoperative therapy with amiodarone and the incidence of acute organ dysfunction after cardiac surgery. Anesth Analg. 1997;85:489-97.

7. Haan CK, Geraci SA. Role of amiodarone in reducing atrial fibrillation after cardiac surgery in adults. Ann Thorac Surg. 2002;73:1665-9. 